Research Paper

\title{
Quantitative Detachment Mechanics of Hydra from Substrates
}

\author{
NEHA KHETAN, SHAGUN MAHESHWARI ${ }^{1}$ and CHAITANYA A ATHALE* \\ Division of Biology, IISER Pune, Dr. Homi Bhabha Road, Pashan, Pune, India
}

(Received on 07 May 2018; Revised on 17 September 2018; Accepted on 24 September 2018)

\begin{abstract}
Hydra is a fresh water hydrozoan living as a solitary polyp with a sedentary feeder lifestyle attached to a substrate. In times of food shortage they are reported to detach from their substrate and move either by drifting or 'somersaulting'. However, the quantification of the mechanical strength of the adhesion of Hydra has not been reported before. Here, we measure the force required to detach Hydra vulgaris and Hydra magnipapillata from a surface and the role of nutritional state and substrate rigidity. For detachment force measurement, we have developed a calibrated flow system, based on a syringe pump. We find the detachment shear stresses are similar whether the animal is well-fed or starved in the two species tested - $H$. vulgaris and $H$. magnipapillata. On the other hand, adhering to a hard substrate like a glass cover slip requires more force to detach $H$. vulgaris as compared to a soft substrate like polyacrylamide gel. Detachment stresses also differ across the two species in the same state. Taken together, it suggests that mechanics of the substrate and ambient flows in the water body could affect passive locomotion of Hydra, while suggesting the magnitude of muscle-based forces required to actively detach it from the substrate.
\end{abstract}

Keywords: Hydra; Laminar Flow Chamber; Drag Force; H. vulgaris; H. magnipapillata

\section{Introduction}

Aquatic life forms, ranging from single-celled to multicellular have evolved a variety of strategies to remain static through adhesion to substrates. The specific mechanism by which they achieve this adhesion ranges from suckers and nanometer scale spatulae to biological adhesives (reviewed by Gorb, 2008). Amongst aquatic animals, the adhesion of the mussel Mytilus edulis has been particularly well studied (reviewed by Waite 2002). The mussel shells attach to rocky substrates with byssal threads consisting of multiple proteins, each contributing distinct mechanical properties (Lin et al., 2007). The amino acid 3,4dihydroxy-L-phenylalanine (dopa) is considered a vital component for the mechanical and chemical properties of adhesive, with each molecule of dopa-enriched peptide requiring on an average $805 \mathrm{pN}$ for dissociation from the substrate (Lee et al., 2006).

Hydra on the other hand, are fresh water dwelling Hydrozoans of phylum Cnidaria that live as

*Authorfor Correspondence: E-mail: cathale@iiserpune.ac.in

${ }^{1}$ Present address: Applied Materials, Santa Clara, California, USA solitary polyps, typically found attached to substrates like stems, branches or leaves under-water. Renewed interest in Hydra is due to its regenerative ability, along with genome sequence and the evolutionary relatedness of the regenerative pathways to vertebrates (Fujisawa, 2006; Watanabe et al., 2009). In their natural environment, Hydra are subjected to gentle flows and so far their movement has been attributed to passive drifting. Wagner has noted that the resistance of an attached Hydra to water flows might be an adaptation to the diverse environmental conditions (still and flowing water) that it is exposed to and its inability to actively swim, once suspended in water (Wagner 1905). The movement of Hydra that are already attached to a substrate was observed to occur by 'somersaulting' - animals attach their tentacles to a new position, detach the basal disk with body contraction, straighten and reattach the basal disk at a new position near the hypostome (Wagner, 1905). A report on Indian samples of Hydra vulgaris described the ability of these animals to actively 
'crawl', a motion that involved 'somersaulting' (Annandale, 1911), similar to that reported earlier. The movement is thought to enable the individual to leave unfavourable environments. The combination of contraction by ectodermal longitudinal muscles and extension by endodermal circular muscles has been used to explain the 'somersaulting' motion. However, a major step in the process necessarily involves the detachment of base. To our knowledge, the mechanics of this detachment is yet to be addressed.

In its sedentary mode, Hydra sp. is attached by its basal-body; also called the basal disk or 'foot' to the substrates by a glandular secretion (Brien, 1960). Like other parts of Hydra, the animal can also regenerate the basal disk when amputated (Amimoto et al., 2006). Histologically, the cells of the disk consist of an inner endoderm and an outer ectoderm. The cells are glandular, conical in shape and filled with granules (Bode et al., 1986). These cells secrete large amounts of mucus needed for attachment of the animal to substrates. The basal disk was thought for long to be a closed structure but more recently a porelike structure in the disk called aboral pore (Shimizu et al., 2007) has been found. While the histology of the foot is understood, the nature of mucus as a bioadhesive, which works under water could be interesting, both from a fundamental perspective of adhesives, as well as applications in biocompatible materials (Waite, 2002). Recently, the glue from Hydra magnipapillata has been characterised and found to be based on glycans and glycoproteins (Rodrigues et $a l .$, 2016a). Additional gene-expression analysis has revealed 21 transcripts to be expressed in the basal disk alone (Rodrigues et al., 2016b). While remaining attached is important for Hydra, movement requires the ability to detach and is equally important. However, till date no quantitative measurements of the detachment force of Hydra has been performed.

Measuring the force for detachment of larger animals, such as Mytius sp. has involved mechanical spring-based instruments (Bell and Gosline, 1996; Denny, 1987), while sea anemone detachment has been measured using force transducers (Koehl, 1977). Such instruments, however do not mimic the naturally occurring flows that aquatic animals are likely to experience and measurements could suffer from artefacts arising from contact. Flow chambers address some of these shortcomings and have been used to study biofouling using turbulent (Schultz et al., 2000) or pumped flows coupled to inline flow meters (Neal et al., 1996). Flow tanks that have been described for whole organism studies (Vogel and LaBarbera, 1978) and parallel-plate flow chambers used for studying leukocyte adhesion (Chen and Springer, 1999) are successful means to measure detachment dynamics of samples, ranging from cells to whole organisms.

We have chosen to characterise the biomechanics of Hydra sp. detachment with calibrated fluid flows, from which we estimate the drag force required to displace the animal from a substrate. We use this device to measure the flow rate required to detach the Hydra from substrates of different stiffness and proceed to examine the role that starvation and substrate stiffness play in the stress required to detach the individuals.

\section{Materials and Methods}

\section{Growth and handling of Hydra}

Hydra vulgaris Ind-Pune (Reddy et al., 2011) and Hydra magnipapillata were obtained from ARI (Pune, India). They were maintained in $\sim 200 \mathrm{ml}$ of "M" solution containing $0.1 \mathrm{mM} \mathrm{KCl}, 1 \mathrm{mM} \mathrm{NaCl}, 1$ $\mathrm{mM} \mathrm{CaCl} \cdot 2 \mathrm{H}_{2} \mathrm{O}, 1 \mathrm{mM}$ Tris $(\mathrm{pH} 8)$ and $0.1 \mathrm{mM}$ $\mathrm{MgSO}_{4} \cdot 7 \mathrm{H}_{2} \mathrm{O}$ in water (Sugiyama and Fujisawa, 1977). The animals were maintained at $18^{\circ} \mathrm{C}$ in an incubator with a lamp, with timer kept on for $\sim 12 \mathrm{~h}$ to artificially induce day-night cycles (Thermo Scientific, USA) and the beaker cleaned on a daily basis. Hydra were fed two to three hatched Artemia sp. (brine shrimp) every two days. Brine shrimps were grown in a $0.6 \%$ saline solution and filtered after washing in tap water before being used as feed.

\section{Imaging and Microscopy}

Individual $H$. vulgaris and $H$. magnipapillata animals were placed in a petri dish with a drop of ' $M$ ' solution to prevent desiccation and imaged using a Leica dissection microscope S8 APO illuminated with a Leica (24 DC) LED control unit and equipped with an EC 3 camera (Leica Microsystems, Germany). The onset of turbulence was recorded using autofocus and automatic exposure settings in video mode on a Canon EOS 1000D camera (Canon Inc., Japan). 
(A)

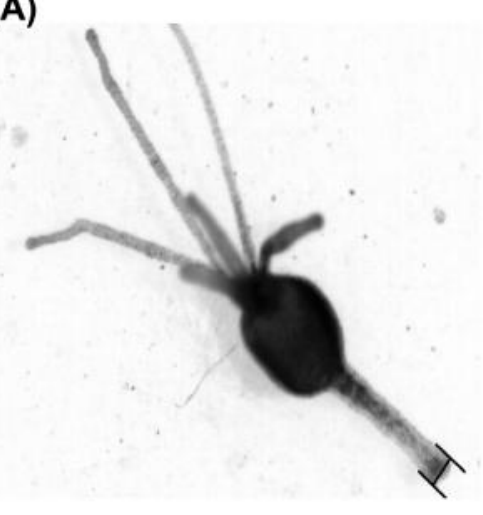

$0.274 \mathrm{~mm}$
(B)

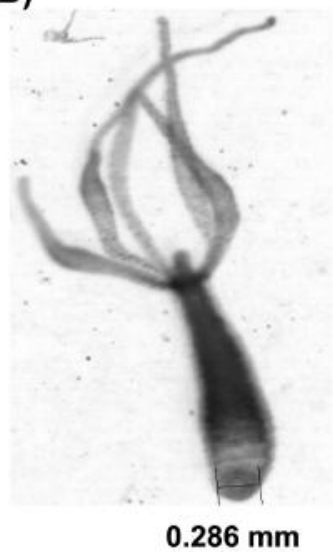

(C)

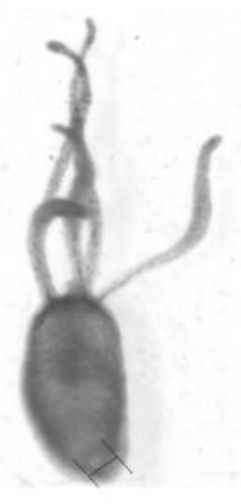

$0.257 \mathrm{~mm}$
(D)

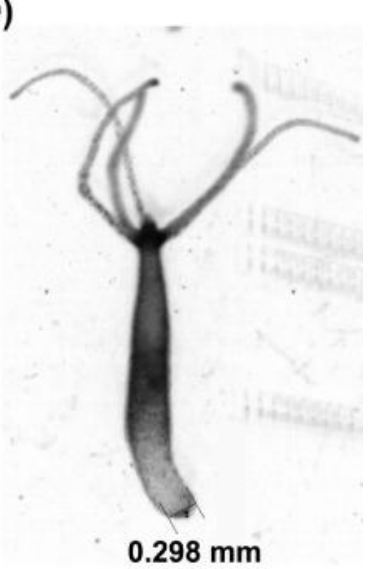

(E)

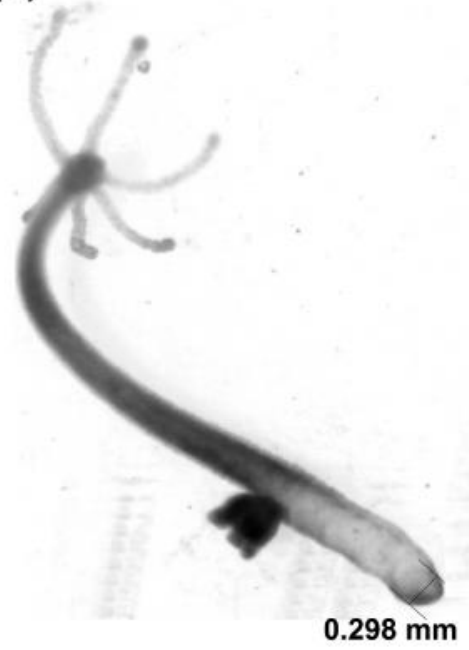

(F)

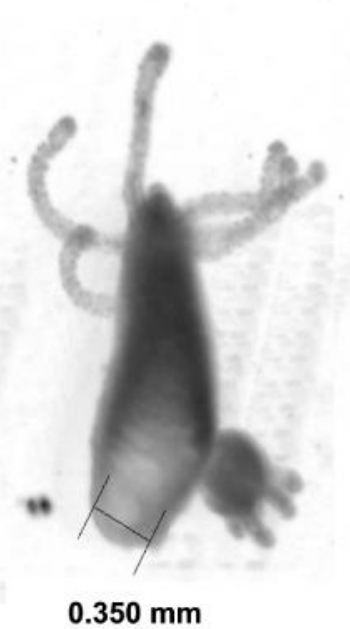

(G)

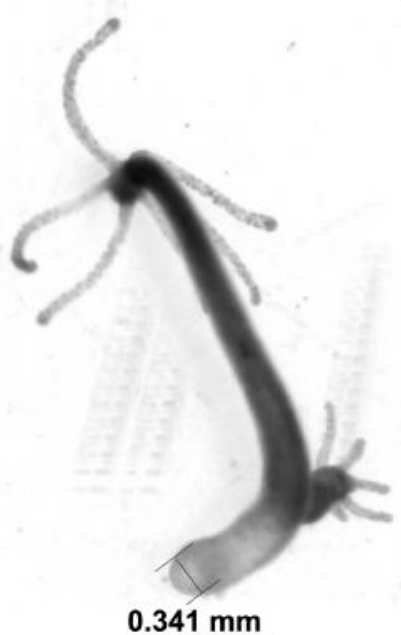

(H)

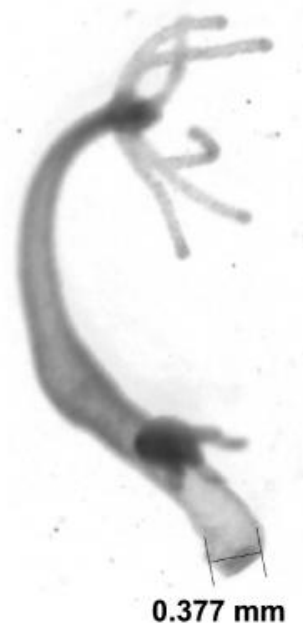

Fig. 1: Estimating the size of Hydra. Images of live animals of (A)-(D) Hydra vulgaris and (E)-(H) Hydra magnipapillata were acquired under a dissection microscope in a drop of "M" solution. The base diameter is marked at the measured site in millimeters (mm). Mean diameters $H$. vulgaris $0.279 \mathrm{~mm}$ and $H$. magnipapillata $0.342 \mathrm{~mm}$

\section{Flow Chamber and Detaching Hydra}

A syringe pump (PhD Ultra, Harvard Apparatus, USA) with a $20 \mathrm{ml}$ plastic syringe (BD Biosciences, India) was connected to polycarbonate tubing of inner diameter (I.D.) $3 \mathrm{~mm}$ (BioRad, USA) and further connected with an adaptor (BioRad, USA) to a tube of I.D. $0.8 \mathrm{~mm}$, taped to the bottom of a glass trough (Fig. 2A). For Hydra detachment experiments, the animals were allowed to attach to a glass coverslip (MicroAid, Pune, India), such that the animal was at a distance of $0.5 \mathrm{~cm}$ from the tube outlet, in line with the fluid flow. Flow experiments typically involved increasing the flow rate from $10 \mathrm{ml} / \mathrm{min}$, with increments of $\sim 2 \mathrm{ml} / \mathrm{min}$ until the animal detached due to the force generated. Those experiments in which the Hydra was either attached to the substrate with its tentacles, or did not attach at all, or failed to detach at all flow rates were ignored in analysis of detachment shear stress. The sample sizes for each animal and condition ranged from 7-10 animals (Table 1).

Table 1: The sample sizes of Hydra sp. detachment under different conditions

\begin{tabular}{lccc}
\hline Organism & Substrate & $\begin{array}{c}\text { Feeding } \\
\text { status }\end{array}$ & $\begin{array}{c}\text { No. of } \\
\text { individuals }\end{array}$ \\
\hline Hydra vulgaris & Glass & Fed & 10 \\
& & Unfed & 10 \\
& Gel & Fed & 7 \\
Hydra magnipapillata & Glass & Unfed & 10 \\
& & Fed & 10 \\
& & Unfed & 7 \\
\hline
\end{tabular}




\section{Modulating Substrate Stiffness}

For experiments to measure the effect of substrate stiffness, a $0.75 \mathrm{~mm}$ thick $5 \%$ polyacrlyamide gel was prepared using a $5 \mathrm{ml}$ solution of $5 \%$ acrylamide and $0.22 \%$ bisacrylamide, 25 ul APS ( $1 / 200$ volume) and 2.5 TEMED (1/2000 volume) (all reagents SigmaAldrich, Mumbai, India) and curing for $15 \mathrm{~min}$ between two plates was layered with water in a standard polyacrylamide gel electrophoresis (PAGE) setup (BioRad, USA). This gel of stiffness of $8 \mathrm{kPa}$ (Tse and Engler, 2010), was layered on the coverslip and the Hydra was allowed to attach to the gel. The remainder of the experiment was performed in a manner similar to the experiments for detaching Hydra from glass coverslips.

\section{Data Analysis}

Images of Hydra and the onset of turbulence were processed using Image J (Schneider et al., 2012). Fitting data to functions was performed using the nonlinear fitting tool (nlinfit) in MATLAB (Mathworks Inc., MA, USA). Statistical testing of mean shear stresses was performed using a two-sided Kolmogorov-Smirnov (KS) test with $95 \%$ confidence interval.

\section{Results}

\section{Morphometry of Hydra and Profile in Flow}

In order to estimate the forces exerted by flow, we needed to characterise the shape and size of Hydra used in this study. Two species were chosen due to the differences in sizes and availability, namely $H$. vulgaris and $H$. magnipapillata. While qualitatively in a dissection microscope $H$. vulgaris was seen to be shorter than $H$. magnipapillata, their widths appeared comparable (Fig. 1). This was confirmed by estimating the mean cross-sectional diameter of the foot as $0.279 \mathrm{~mm}$ for Hydra vulgaris (Fig. 1AD) and $0.342 \mathrm{~mm}$ for $H$. magnipapillata and height as 3 and $5 \mathrm{~mm}$ respectively (Fig. 1E-H). Given the base of the hydra is approximately circular, we calculate the average disk area $\left(A_{d}\right)$ of $H$. vulgaris to be $0.24 \mathrm{~mm}^{2}$ and $H$. magnipapillata to be 0.37 $\mathrm{mm}^{2}$.

\section{Laminar Flow in Chamber and Positioning Hydra}

The flow chamber set-up consists of a syringe pump connected by tubing to a trough filled with medium. The Hydra are submerged in the medium and subjected to the flow (Fig. 2A). We proceeded to characterise the nature of fluid flow in the chamber, in order to test whether the forces generated are due to laminar flows.

To this end, the experiment was performed in absence of any obstacles to characterise the syringe pump driven water jet. Safranin dye was added to the water in the syringe in order to provide contrast for visualizing the flow as it entered the trough. We observed turbulence in the flow at a distance $L_{t}$ from E, the exit from the pipe (Fig. 2B). The point of turbulence, along the length of the flow was marked as T. The graph of $L_{t}$ as a function of flow rate (Q) shows that even for the fastest flow-rates, the eddies begin $1 \mathrm{~cm}$ from the pipe exit, E (Fig. 2C).

In order to understand the quantitative nature of the flow rate dependence of the length of turbulence onset, we attempted to empirically model this data based on the theory of turbulence due to a moving fluid entering a static body of the same fluid. We fit the data of $L_{t}$ with changing $\mathrm{Q}$ by modifying the standard expression for Reynolds number (Re). By definition, $\operatorname{Re}=(\rho \cdot L \cdot V) / \eta$, where, $\rho\left(\mathrm{kg} \cdot \mathrm{m}^{-3}\right)$ is the density of fluid, $L(m)$ is the characteristic length of the flow, $V\left(m \cdot s^{-1}\right)$ is the fluid velocity and $\eta\left(N \cdot s \cdot m^{-2}\right)$ is the dynamic viscosity. By dimensional analysis, the volume flow rate $Q$ with units $m^{3} \cdot s^{-1}$, is related to the fluid velocity by:

$$
V=Q / A_{f}
$$

where, $A_{f}\left(\mathrm{~m}^{2}\right)$ is the area of cross section of the fluid flow. Substituting for V and based on the fact that $R e, \rho, \eta$ and $\mathrm{A}$ are constant for a given flow rate, we invoke a lumped constant $c_{1}=(\operatorname{Re} \cdot A \cdot \eta) / \rho$. The characteristic length at the point of turbulence is the length of turbulence onset, $L_{t}$. As a result, we arrive at the following expression:

$$
L_{t}=c_{1} / Q
$$

which we proceed to fit to the experimental data in Fig. 2C. The fit value of $c_{1}$ is $1.06 \mathrm{~cm}^{4} / \mathrm{s}$. Based on this fit, $L_{t}$ is always greater than $1 \mathrm{~cm}$ for all values of the flow rate $\mathrm{Q}$ that were used in experiment.

For comparison, we used results from environmental fluid mechanics, where the distance 


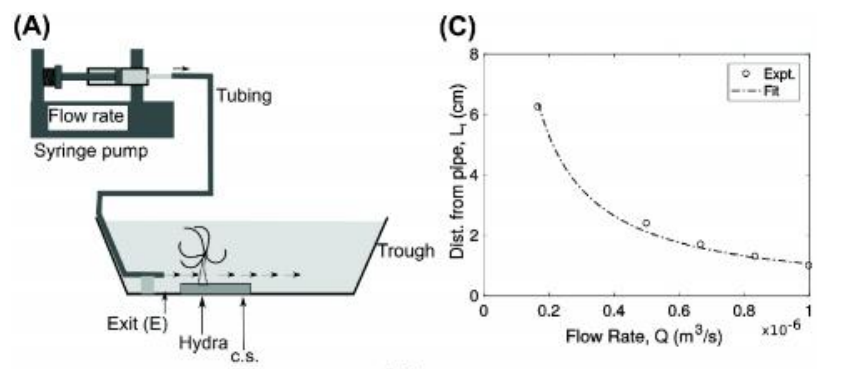

(B)

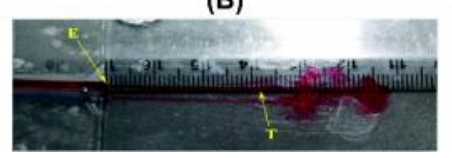

Fig. 2: Detachment of Hydra in a laminar flow device. (A) A schematic representation of the experimental set-up with Hydra placed on a coverslip (c.s.) at a fixed distance from the pipe exit (E) submerged in buffer in a glass trough. The arrows indicate direction of flow of water from the syringe pump. (B) The view from the top of distance of the onset of turbulence (T) flow from the pipe exit (E) estimated by flowing safranin containing water. (C) The turbulence length $\left(L_{t}\right)$ is plotted as a function of the flow rate, $Q$ (circle) and fit (dashed line) by the equation $L_{t}=c_{1} / Q$ (Equation 2)

before the onset of spreading of the flow in an analogous system to ours, is also referred to as the 'virtual source' distance from the exit nozzle and is $5 r$, where, $r$ is the radius of the pipe (Cushman-Roisin, 2006). The tubing used for the flow device has an inner diameter of $0.3 \mathrm{~cm}$, resulting in a virtual source distance of $0.75 \mathrm{~cm}$ from the nozzle. Based on these results, we placed Hydra at a distance of $0.5 \mathrm{~cm}$ from $E$ in subsequent experiments, to ensure that the force experienced is due to laminar flows alone.

\section{Relating Drag Force Experienced by Hydra to the Flow Rate}

Since Hydra is an aquatic animal, we used the flow of water to generate force to detach it, since it avoids potential artefacts of mechanical attachment of the organism to a cantilever. The fluid drag force experienced by sessile animals in a moving fluid has been previously described by Vogel (Vogel, 1996), through the standard drag equation based on Bernoulli's principle. For sessile animals in a flow, it has been shown to depend on the frontal projecting area of the animal with simplifications of the animals to standard geometric forms. We have therefore assumed a simplified geometry for Hydra - a cylinder. Further, the section of the cylinder experiencing the flow (half the area of the cylindrical section) then becomes the projected area $\left(A_{p}\right)$ as seen in in Fig. $3 \mathrm{~A}$. Then, drag-force $\left(F_{d r a g}\right)$ experienced by the Hydra can be estimated by:

$$
F_{\text {drag }}=C_{d} \cdot \rho \cdot V^{2} \cdot A_{p} / 2
$$

where, $C_{d}$ is the drag coefficient, $\rho$ is the density of the fluid, $V$ is the velocity of flow and $A_{p}$ is the projected area of the body in the path of fluid flow, shown schematically in Fig. 3A. On substituting $V$ from Equation 1 into the expression for drag force (Equation 3 ), we obtain an expression of the drag force, in terms of volume flow rate:

$$
F_{\text {drag }}=\frac{C_{d} \cdot \rho \cdot Q^{2} \cdot A_{p}}{2 \cdot A_{f}^{2}}
$$

Thus, the detachment force of Hydra can be calculated using Equation 4, based on two assumptions that justify the use of the V (and in turn Q) values of the jet of water as it exits the pipe:

1. The Hydra are placed at a distance from the pipe that is always less than the distance, where turbulence onset is observed (Fig. 2).

2. Dissipation of the flow due to spreading can be ignored, since the Hydra are placed at a distance less then the 'virtual source' distance.

The drag coefficient $\left(\mathrm{C}_{\mathrm{d}}\right)$ is a dimensionless constant and depends on properties of the object and fluid such as shape and Reynolds number. For our calculations, choice of $\mathrm{C}_{\mathrm{d}}$ was made by approximating the shape of Hydra to a cylinder with its long axis normal to the direction of flow. Based on the length:width ratio of Hydra, the drag coefficient of 0.68 was chosen, based on standard results for a cylinder with length to the diameter ratio of $2: 1$ (Stoecker, 2004). For the sake of simplicity, we assumed the shape to be constant for the Hydra across all the conditions.

The calculated estimates of force with increasing flow rate was calculated, based on a function of the form:

$$
F_{d}=c_{2} \cdot Q^{2}
$$


(A)

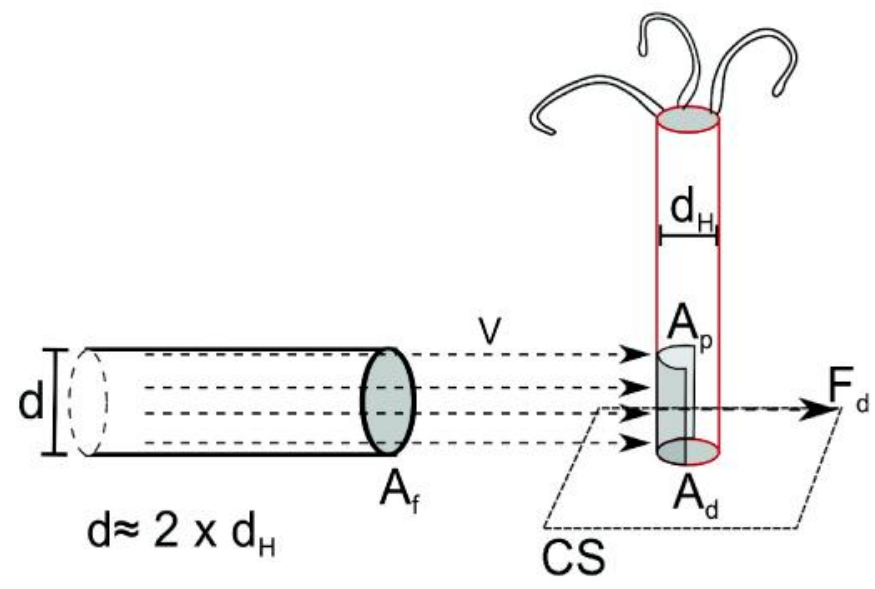

(B)

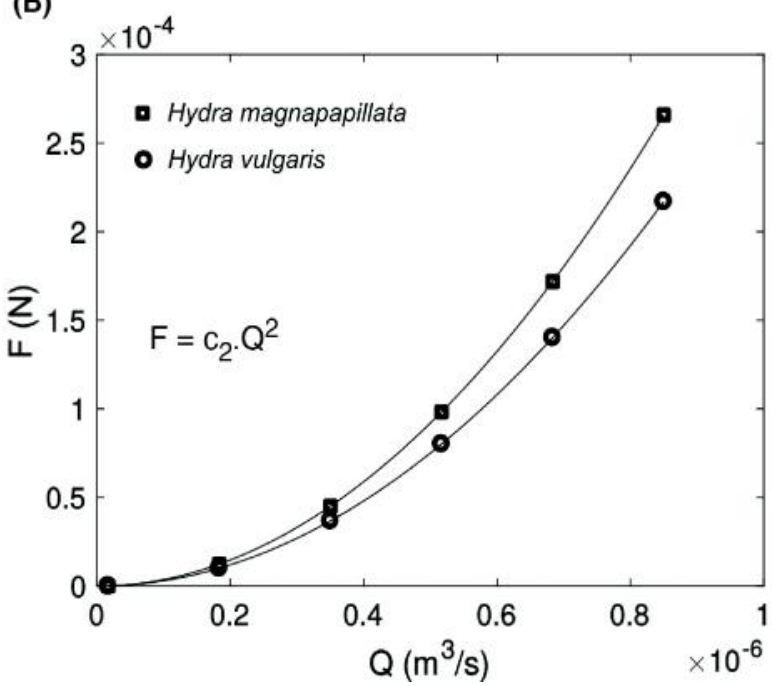

Fig. 3: Forces acting on Hydra. (A) The schematic represents the direction of fluid flow from the exit of the tube with the Hydra experiencing a drag force $\left(F_{d}\right)$ along the direction of flow velocity $(v)$. For simplicity, the animals are assumed to be in an upright orientation with a simplified cylindrical geometry. $A_{f}$ : flow cross-sectional area; $A_{p}$ : projected area of the Hydra in flow, $A_{d}: A_{d}$ disk area of Hydra, d: inner diameter of pipe and CS: coverslip. From measurements $A_{f} \approx 2 A_{d}$ (B) The drag force experienced by Hydra due to flow is calculated from Equation 5 with the constant c2 calculated for $H y d r a$ vulgaris to be $3 \times 10^{8} \mathrm{~kg} \mathrm{~m}^{-5}$ (circles) and for Hydra magnipapillata to be $3.68 \times 10^{5} \mathrm{~kg} \mathrm{~m}^{-5}$ (square)

where, $Q$ is the volume flow rate $\left(\mathrm{cm}^{3} / \mathrm{s}\right)$ and $c_{2}=$ $\left(C_{d} \cdot \rho \cdot A_{p}\right) / 2 \cdot A_{p}{ }^{2}$ is a constant that differs for the two species due to the differences in the projected area $A_{p}$. The values of $c_{2}$, obtained by substitution, are $3 \times 10^{8} \mathrm{~kg} . \mathrm{m}^{-5}$ for Hydra vulgaris and $3.68 \times 10^{8}$ $\mathrm{kg} . \mathrm{m}^{-5}$ for Hydra magnipapillata. Based on Equation 5 , we can plot the force for both species across the range of flow rates used experimentally (Fig. 3B). By noting the flow rate at which mechanical detachment of Hydra is observed, we can then estimate the shear force of detachment.

\section{Estimating Detachment Force Variation with Species, Feeding State and Substrate Rigidity}

In order to measure detachment forces, as a first step, individual Hydra were allowed to attach to a glass coverslip (coated or uncoated) in the incubator. At the time of measurement, the coverslip was removed from the incubator and placed at a distance of 0.5 $\mathrm{cm}$ from the pipe exit (E) (Fig. 2A). The flow rate (Q) was gradually increased until the Hydra detached. Measurements were repeated for 10 individuals of $H$. vulgaris and $H$. magnipapillata. By starving one set of animals, we addressed the effect of nutritional state. We also compared the effect of changing substrate stiffness on $H$. vulgaris. For each experimental condition, the flow rate at which Hydra detached was used to calculate the force of detachment (Equation 5). It is reasonable to assume that both species have a circular cross-section. Hence, the area of the basal disk is estimated from the mean diameter of each species. The ratio of the detachment force to the basal disk area then results in shear stress of detachment. H. vulgaris detaches from glass substrates over a wide range of shear stresses, ranging from 0.57 to $2.02 \mathrm{MPa}$, while $H$. magnipapillata detachment shear stresses range between 3 and $3.7 \mathrm{MPa}$ (Fig. 4A). While the difference between detachment shear stress of fed and unfed $H$. vulgaris is not statistically significant, that between substrates of glass and 5\% polyacrylamide gel is significant, for the same species in the same nutritional state. However, the shear stress required to detach $H$. magnipapillata is higher than that for $H$. vulgaris in the same nutritional state, while the animals are attached to glass. All comparisons were based on a two-sided Kolmogorov-Smirnov test, with $95 \%$ confidence interval. Based on our measurements, the detachment shear stress is estimated to be of the order of $10^{2}-10^{3} \mathrm{~N} / \mathrm{m}^{2}$, which is two orders of magnitude, smaller than in molluscs (Denny, 1987). This suggests that the Hydra adhere 
(A)

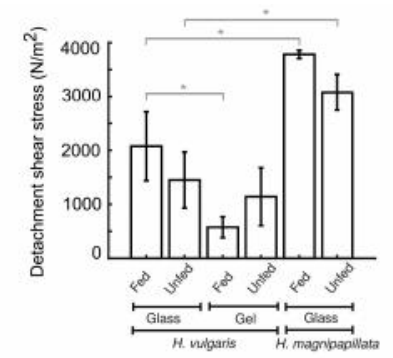

(B)

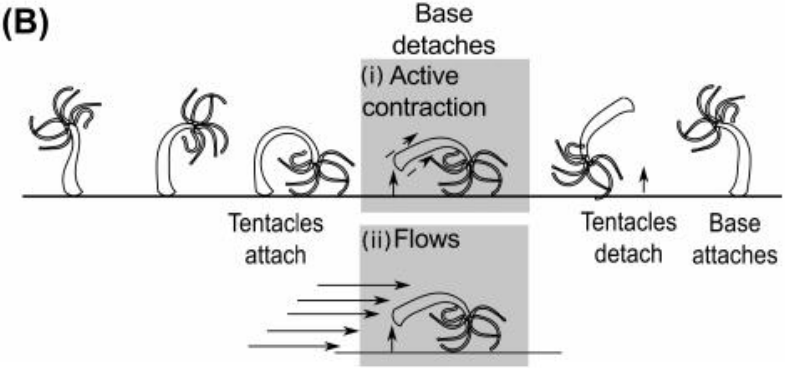

Fig. 4: Detachment shear stress. (A) The mean shear stress ( \pm s.e.m.) of detachment for $H$. vulgaris and $\boldsymbol{H}$. magnipapillata under fed and unfed conditions for individual hydra attached to glass (stiffness $7.29 \mathrm{x}$ $\left.10^{7} \mathrm{kPa}\right)$. $\mathrm{H}$. vulgaris detachment was also tested on a $5 \%$ polyacrylamide gel (stiffness $\sim 8 \mathrm{kPa}$ ). The mean shear stress was compared, using a two-sided Kolmogorov-Smirnov test at $\alpha=0.05$ (*). (B) The schematic depicts the stages in 'somersaulting' movement by Hydra. The stage of detachment of base from the substrate can be driven either by (i) active contraction along the body-length or (ii) the force generated by flows in the environment

to their substrate, with a weak glue in a manner that depends on the substrate-stiffness and the species.

\section{Discussion}

Here, for the first time we have quantitatively characterised the mechanics of Hydra detachment from an attached position on a substrate. We use a simple flow device which generates known amounts of shear stress through flows generated in the aqueous. We can show that smaller shear stresses are required to detach $H$. vulgaris from soft as compared to hard substrates. We find that the shear stress of detachment between fed and unfed states shows no statistically significant difference for the same substrate of both $H$. vulgaris and $H$. magnipapillata.

The steady attachment of sedentary aquatic animals can occur by multiple mechanisms, but the strength of attachment to the substratum is related to its behaviour, as well as the flows in which it lives (Koehl, 1977; Dodou et al., 2011; Ditsche and Summers, 2014). Typically, free flowing streams with a gentle flow are reported to have flow speeds in the range of $0.5 \mathrm{~m} / \mathrm{s}$ to $3 \mathrm{~m} / \mathrm{s}$. We can make an order of magnitude estimate of the shear stress $(\mathrm{S})$ based on the fluid drag-force $\left(F_{d r a g}\right)$ from Equation 3 which simplifies to $S=\rho \cdot v^{2} / 2 \cdot r$ using the $C_{d}$ of 0.68 based on the 2:1 ratio of length to the diameter (Stoecker, 2014) of Hydra and assuming the Hydra can be treated as cylinders, so the projected half-area of the cylinder is affected by drag. Based on an estimate of the projected areas of the two species, H. vulgaris will be expected to experience shear stresses between $1.8 \times 10^{3}$ and $6.6 \times 10^{4} \mathrm{~N} / \mathrm{m}^{2}$, while H. magnipapillata is expected to experience between $2.5 \times 10^{3}$ and 8.9 $\mathrm{x} 10^{4} \mathrm{~N} / \mathrm{m}^{2}$. Given that we measure detachment shear stresses for both species, ranging between to $5 \times 10^{2}$ and $3.7 \times 10^{3} \mathrm{~N} / \mathrm{m}^{2}$ (Fig. 4A), it would suggest normal flows that the animal is likely to experience, would be sufficient to detach the animal from the substrate. This would suggest, that in addition to active motion, Hydra can also be passively detached from its substrate. This is corroborated by observations of drifting animals found in their natural habitat. Careful observations in still and moving streams combined with measurements of flow-rates in situ could be potentially used to test this prediction.

The measurements we report here are made on two kinds of artificial substrates-glass and 5\% polyacrylamide gel. While glass is very stiff with a Young's modulus of $72.9 \mathrm{MPa}$, the gel used has a reported stiffness of $8 \mathrm{kPa}$ (Tse and Engler, 2010). Our measurements suggest that Hydra vulgaris is less firmly attached on a soft substrate, as opposed to a hard substrate. The comparison with the bulk modulus of elasticity of freshwater aquatic plant leaves, which ranges between 1 and $10 \mathrm{MPa}$ (Touchette et al., 2014), would suggest our measurements cover the range of stiffness that Hydra could be expected to encounter when attached to leaves. While on the one hand, the differences in detachment are less than an order of magnitude and subject to large variations, it would be interesting in future to systematically vary substrate stiffness and examine the role it plays in movement of the animal. Additionally, the mechanical properties of specific plants and other objects to which Hydra is naturally found attached to, could also determine whether there 
is any role at all for the substrate stiffness.

In our experiments, we have used inert substrates during the detachment measurements, in order to study the role of mechanical properties in the absence of any material properties. However, it could have been possible that the chemical nature of the 'glue' might also be modulated during detachment, through hydrolysis by some enzyme produced in the animal itself. However, a recent study that investigated the glue concluded that active contraction is more likely to be the primary method by which Hydra achieves detachment (Rodrigues et al., 2016a). Since we do not observe a clear starvation dependent weakening of the bond, our data corroborates the potential role of active contraction. A potential limitation of the current study is that we only observed whether the animal has detached from its base (endpoint), since we do not record the motion prior to it's detachment. However, even if it were to perform 'somersaulting' locomotion during the measurement, the fact that, the flow diameter is approximately $1 / 10$ the length of the Hydra (Fig. 3A) suggests the validity of our approach. In future, the predictions of either 'somersaulting' or passive detachment of Hydra could be tested by motion-capture videography to capture the entire cycle of movement (Fig. 4B).

The force required to detach Hydra is two orders of magnitude smaller than the detachment stress of reported for the well studied sedentary marine mussel Mytilus sp. (Denny, 1987). We hypothesise that the difference in habitat of Hydra sp. which mostly inhabits ponds and slow-flowing streams means that the detachment stresses do not need to be as high as those observed in Mytilus mussels, typically found attached to inter-tidal rocks subject to constant wave action (Bell and Gosline, 1996). Based onreports by Annandale and others, it is reasonable to assume this weaker adhesion of Hydra is an adaptation to the forces generated by currents it usually experiences in its natural habitat and for the mode of motility that it adopts.

The measurement setup, while simple, provides useful initial answers to a mechanical approach to animal behaviour. Potentially, in future, higher flow rate methods would require taking into consideration the turbulent regime (Schultz et al., 2000). In addition, the starvation conditions in the native environment that trigger 'somersaulting' movement are not clearly reported. In our work, we have empirically chosen a week of starvation. In future a controlled study on the factors and duration of nutrient withdrawal, combined with mechanics could help us better understand the triggers that govern the decision of Hydra to move.

In conclusion, we have characterised the shear stress of detachment of two species of Hydra and find them to range between 0.5 and $3.7 \mathrm{kPa}$. The detachment stress is independent of the nutritional state (i.e. fed as compared to starved for one week) and shows a small change with substrate-stiffness. In addition, we find that $H$. magnipapillata is detached at a higher stress value, as compared to $H$. vulgaris. Based on its natural environment, where the animal is typically found attached to the under side of leaves in ponds and lakes, it would appear that ambient flows by themselves could generate comparable shear stresses of detachment. During 'somersaulting' locomotion, the forces required to be generated by the muscles will also necessarily need to be of comparable magnitude as the detachment stress reported here. This sets the stage for a more quantitative understanding of both passive and active modes of locomotion of Hydra in its natural habitat.

\section{Author contributions}

NKperformed the calculation, made the figures and wrote the manuscript SM assembled the device, performed the measurements and acquired the images CAA conceptualized the study, made the figures, wrote the manuscript and supervised the study.

\section{Acknowledgements}

We would like to acknowledge the kind gift of Hydra magnipapillata and Hydra vulgaris by Sanjeev Galande and help from Girish Ratnaparkhi and Surendra Ghaskadbi in culturing the animals.

\section{Competing interests}

The authors declare they have no competing interests.

\section{Funding}

This work was funded by IISER Pune core funding. NK was funded by a fellowship from CSIR (09/ 936(0128)/2015-EMR-1). 


\section{References}

AmimotoY, Kodama R and Kobayakawa Y (2006) Foot formation in Hydra: a novel gene, anklet, is involved in basal disk formation Mech Dev 123 352-361

Annandale N (1911) Freshwater Sponges, Hydrozoids and Polyzoa. Taylor and Francis Bell E C and Gosline J M (1996) Mechanical Design of Mussel Byssus : Material Yield Enhances Attachment Strenght J Exp Zool 199 10051017

Bode H, Dunne J, Heimfeld S, Huang L, Javois L, Koizumi O, Wester-field J and Yaross M (1986) Transdifferentiation Occurs Continuously in Adult Hydra Curr Top Dev Biol 20 257-280

Brien P (1960) The fresh-water hydra American Scientist 48 348A 461-475

Chen S and Springer T A (1999) An automatic braking system that stabilizes leukocyte rolling by an increase in selectin bond number with shear J Cell Biol 144 185-200

Cushman-Roisin B (2006) Environmental Fluid Mechanics. Wiley-Blackwell, Hoboken NJ, USA

Denny M W (1987) Lift as a mechanism of patch initiation in mussel beds J Exp Mar Biol Ecol 113 231-245

Ditsche P and Summers A P (2014) Aquatic versus terrestrial attachment: Water makes a difference Beilstein $J$ Nanotechnol 5 2424-2439

Dodou D, Breedveld P, de Winter J C, Dankelman J and van Leeuwen J L (2011) Mechanisms of temporary adhesion in benthic animals Biol Rev Camb Philos Soc 86 15-32

Fujisawa T (2006) Hydra is joining the bandwagon Bio Essays 28 560-562

Gorb S N (2008) Biological attachment devices: exploring nature's diversity for biomimetics Phil Trans Roy Soc A 3661557 1574

Koehl M A R (1977) Effects of Sea Anemones on the Flow Forces they Encounter J Exp Biol69 85-105

Lee H, Scherer N F and Messersmith P B (2006) Single-molecule mechanics of mussel adhesion Proc Natl Acad Sci USA 103 12999-13003

Lin Q, Gourdon D, Sun C, Holten-Andersen N, Anderson T H, Waite J H and Israelachvili J N (2007) Adhesion mechanisms of the mussel foot proteins mfp-1 and mfp-3 Proc Natl Acad Sci USA 104 3782-3786

Neal A, Simoes F and Yule A (1996) Interactions between shear rates and biofilms affecting exploratory behaviour by cyprids ofelminius modestus (cirripedia) Marine Biology 127 241-246
Reddy C P, Barve A and Ghaskadbi S (2011) Description and phylogenetic characterization of common hydra from India Curr Sci 101 736-738

Rodrigues M, Leclère P, Flammang P, Hess M W, Salvenmoser W, Hob-mayer B and Ladurner P (2016) The cellular basis of bioadhesion of the freshwater polyp hydra $B M C$ Zoology 13

Rodrigues M, Ostermann T, Kremeser L, Lindner H, Beisel C, Berezikov E, Hobmayer B and Ladurner P (2016) Profiling of adhesive-related genes in the freshwater cnidarian Hydra magnipapillata by transcriptomics and proteomics Biofouling 32 1115-1129

Schneider C, Rasband W and Eliceiri K (2012) NIH Image to Image J: 25 years of image analysis Nature Methods 9671 675

Schultz M P, Finlay J A, Callow M E and Callow J A (2000) A turbulent channel flow apparatus for the determination of the adhesion strength ofmicrofouling organisms Biofouling 15 243-251

Shimizu H, Takaku Y, Zhang X and Fujisawa T (2007) The aboral pore of hydra: evidence that the digestive tract of hydra is a tube not a sac Dev Genes Evol 217 563-568

Stoecker H (2004) Taschenbuch der Physik, 5 edn, Verlag Harri Deutsch, Frankfurt, Germany

Sugiyama T and Fujisawa T (1977) Genetic Analysis of Developmental Mechanisms in Hydra I. Sexual Reproduction of Hydra Magnipapillata and Isolation of Mutants Dev Growth and Differ 19 187-200

Touchette B W, Marcus S E and Adams E C (2014) Bulk elastic moduli and solute potentials in leaves of freshwater, coastal and marine hydrophytes. Are marine plants more rigid? AoB Plants 6 plu014

Tse J R and Engler A J (2010) Preparation of hydrogel substrates with tunable mechanical properties. In: Curr Prot Cell Biol, Chapter 10: Unit 10.16, Wiley

Vogel S (1996) Life in Moving Fluids: The Physical Biology of Flow, 2 edn. Princeton University Press, NJ, USA

Vogel S and LaBarbera M (1978) Simple Flow Tanks for Research and Teaching Bio Science 28 638-643

Wagner G (1905) Memoirs: On Some Movements and Reactions of Hydra J Cell Sci s2 585-622

Waite H J (2002) Adhesion a la Moule Integr Comp Biol 42 11721180

Watanabe H, Hoang V, Matner R and Holstein T (2009) Immortality and the base of multicellular life: Lessons from cnidarian stem cells Semin Cell Dev Biol 20 11141125 . 